\title{
A Preliminary Study of the Effects from Different Sources of Qi on Telomeres
}

\author{
Zhongzhen Cai ${ }^{1 \#}$, Yu Chen ${ }^{1 \#}$, Lin Wang ${ }^{1}$, Jie Teng ${ }^{1}$, Yuming Chen ${ }^{1}$, Mengmei Li ${ }^{1}$, Qian Feng ${ }^{1}$, Caixia \\ $\mathrm{Li}^{1}$, Glen Rein ${ }^{2}$, B. Qing Tang ${ }^{1}$ and Xuemei Bai ${ }^{1 *}$ \\ ${ }^{1}$ ENNOVA Institute of Life Science and Technology, ENN Group, Langfang 065001, China \\ ${ }^{2}$ Quantum Biology Research Lab, PO Box 1402, Ridgway, CO, USA
}

\#These authors are equally contributed to this work

*Corresponding author: Xuemei, ENNOVA Institute of Life Science and Technology, ENN Group, Langfang 065001, China

\section{ARTICLE INFO}

Received: 幽 December 02, 2020

Published: 慧 January 20, 2021

Citation: Zhongzhen Cai, Yu Chen, Lin Wang, Jie Teng, Yuming Chen, Mengmei Li, Qian Feng, Caixia Li, Glen Rein, B Qing Tang, Xuemei. A Preliminary Study of the Effects from Different Sources of Qi on Telomeres. Biomed J Sci \& Tech Res 33(2)2021. BJSTR. MS.ID.005374.

Abbreviations: TCM: Traditional Chinese Medicine; RPV: Rotating Poynting Vector; TERT: Telomere Reverse Transcriptase; TERC: Telomerase RNA Component; FBS: Fetal Bovine Serum; SD: Standard Deviation; ROS: Reactive Oxygen Species

\section{ABSTRACT}

The concept of a life force energy called "Qi" is a fundamental concept in Traditional Chinese Medicine (TCM), despite the fact that it can not be measured using modern technology. For this study, we selected three different forms of potential sources of Qi to study the effect of Qi on cellular and organ levels using telomeres as an indicator of aging. The three types of sources of Qi are as follows: The external Qi from a Qigong master, internal Qi generated by ingesting the Qi-invigorating Chinese herb, Astragali Radix, and from a Rotating Poynting Vector (RPV) device which generates an RPV-field which is reported to have similar property as Qi [1]. The results showed that for cells, the expression of TERT gene was up regulated and the telomere length was prolonged after the treatment of different Qi, but the change of telomerase activity was not significant, with only a slight increase at 4 hours after the treatment of Qigong external Qi. For animal organs, Qi from Astragali Radix was found to prolong telomere length in the heart, liver, spleen and lung tissues, but not in the kidney, brain or skeletal muscle of mice. We preliminarily conclude that the three different sources of Qi showed some similarity at the cellular level to improve the status of aging and that Qi-invigorating herbs can delay the shortening of telomere for selected organs of mice. More research needs to be conducted to better understand the effect of Qi on aging as well as its biochemical mechanisms.

Keywords: Qi; Qigong External Qi; Qi-Invigorating Herb; Telomerase; Telomere Length; TERT Gene

\section{Introduction}

"Qi" (pronounced chee) is the most basic and important concept in traditional Chinese medicine (TCM), and it is considered the driving force for all life-sustaining activities. In Bian Que's "Difficulties Sutra", it is said that "Qi is fundamental to all human beings". Zhuang Zi's "Knowledge Rambling in the North" also said "Human's life is the gathering of Qi. While Qi gathering means to be alive, Qi dispersing means to die." In TCM clinical practices, Qi is one of the most commonly used indicators for evaluating health conditions. In the past 30 years, many researchers have tried to illuminate the nature of Qi from different points of view. Wallace et al. [2,3] proposed that mitochondria are related to the source, form, and function of Qi as well as its pathological associations and thus speculated a link between Qi and mitochondria. Based on experimental phenomena such as sound wave propagation along a meridian (add reference), calcium ion oscillation in cell membranes and changes in cell activity, Miao Qiang et al. concluded that Qi is a biological wave that fills the human body with its frequencies in the audio range [4]. Lin Junshan, et al. [5] proposed that Qi is a quantum field as described in modern microscopic physics and pointed out that we need to use quantum mechanical methods to study it [5]. Recently some scholars recognized that there is a close linkage between Qi and Rotating Poynting Vector field (RPV-field, also known as torsion-field) [6]. 
For example, it was reported that the external Qi emitted by a Qigong master has similar properties to an RPV-field [1,6], including:

1) penetrability which cannot be affected by optical or electromagnetic shielding and still produces various strong physical effects (such as: actuation, mutagenesis, imaging, germination, penetration etc.);

2) action at a distance which is not limited by time and space and can break through spatial barriers;

3) can produce instantaneous effects like altering the high-speed rotation of radiation emitted from the body as it stimulates peanut germination and growth;

4) both exist in two opposite forms where the left-handed fields accelerate life processes (such as promoting flower blooming [7]) and right-handed fields reverse life processes (such as protein denaturation in cooked eggs [8]).

In TCM, the relationship between Qi and aging or longevity has long been established. In Western medicine, telomeres are an important biomarker for aging. During somatic cell division, chromosomes lose 50-200 bp of terminal DNA once they replicate [9], referred to as shortening of chromosomal telomeres. In addition, based on the Hayflick Limit theory, once the telomeres are shortened to a certain length, they no longer have the ability to initiate chromosome replication. When chromosomal replication ability is lost, it may lead to aging or even death of the organism [10]. However, the enzyme telomerase can maintain the length of telomeres as we age. This process involves an RNA component (telomerase RNA component, TERC) and a protein component (telomere reverse transcriptase, TERT). Previous studies have shown that overexpression of TERT prevented the shortening of telomeres in normal fibroblasts as well as epithelial cells [11]. Therefore, the study of telomerase activity, telomere length and TERT gene expression may reflect the cellular aging process at physiological, biochemical, and genetic levels. For this study, our objective was to examine the effect of three different sources of Qi on aging of M1 cells using modern indices including the length of telomeres, the activity of telomerase, and TERT gene expression. Then we extended the research to the organ level by examining the effect of Astragali radix, a Qi-invigorating herb, on the three aging related indices in different organs. The significance of this study is two-fold:

1) it explores the similarities of Qi generated from different sources;

2) it provides data to support a relationship between Qi and aging using modern scientific indices.

\section{Materials and Methods}

\section{Herbal Preparation}

The Astragali Radix (short named as A.R.) herb was purchased at a Chinese medicine chain-store (Tongrentang, Beijing), and was used to make an herbal preparation. After chopping $50 \mathrm{~g}$ of the herb into small pieces and soaking them in $500 \mathrm{ml}$ deionized water for an hour, the herb was extracted in boiling water for 30 minutes. The procedure was repeated twice. The pooled extract was dried by evaporating the solvent under reduced pressure, and a water extract of Astragali Radix was obtained with a yield of $14.4 \%$ (w/w).

\section{Cell Culture and Treatment}

M-1 mouse kidney collecting duct cell line was obtained from National Infrastructure of Cell Line Resource (Beijing, China, http:// www.cellresource.cn). The cells were cultured as monolayers in DMEM/F12 medium (1:1) supplemented with 10\% fetal bovine serum (FBS) and incubated in a humidified $5 \% \mathrm{CO}_{2}$ incubator at 37 ${ }^{\circ} \mathrm{C}$. The medium was changed 2-3 times per week. Stock cells were grown in $10 \mathrm{~cm}$ culture dishes and split at a sub-cultivation ratio of $1: 5$.

Application of Qi from Astragali Radix to Cells: For this experiment, M-1 cells were seeded at a density of $5-6 \times 10^{5}$ cells/ plate into $10 \mathrm{~cm}$ plates. After cell attachment for $24 \mathrm{~h}$, for the treatment group, the Astragali Radix extract was dissolved in PBS and added to the culture medium at a final concentration of $50 \mu \mathrm{g} /$ $\mathrm{ml}$. The untreated control group was given PBS only. After $4 \mathrm{~h}$, the culture medium was removed, and fresh medium was added. All cultures were incubated for an additional $48 \mathrm{~h}$. At $4 \mathrm{~h}, 24 \mathrm{~h}$ and $48 \mathrm{~h}$ respectively during the treatment, cells were collected using trypsin-EDTA $(0.25 \%)$ and, centrifuged at $800 \mathrm{~g}$ for $5 \mathrm{~min}$. The cells were then re-suspended in $5 \mathrm{ml}$ of PBS and subjected to the different assays. All experiments were performed in triplicate.

Treatment of Cells by a Qigong Master: In this study, a Qigong master, named Guowei Lin, was invited to apply external Qi to the cells. M-1 cells were seeded at a density of $5 \sim 6 \times 10^{5}$ cells/plate into $10 \mathrm{~cm}$ plates. After cell attachment for $24 \mathrm{~h}$, external Qi was applied from Lin's hands with a distance of 5 10 cm to a set of triplicated plates for $15 \mathrm{~min}$ at room temperature, whereas another set of triplicated control plates were left untreated for $15 \mathrm{~min}$ at room temperature in a separate room. The Qigong master was unaware of the control plates. After the treatment, the plates were sent back to the same incubator for an additional $48 \mathrm{~h}$ incubating. At $4 \mathrm{~h}, 24 \mathrm{~h}$ and $48 \mathrm{~h}$ respectively after the treatment, the cells were collected and subjected to the different assays. All experiments were performed in triplicate.

Treatment of Cells with the RPV-Field: M-1 cells were seeded at a density of $5 \sim 6 \times 10^{5}$ cells/plate into $10 \mathrm{~cm}$ plates. After 
cell attachment for $24 \mathrm{~h}$, plates were placed in the RPV-field for the treatment group, while the control group was left for normal cultivation without RPV-field treatment. All the plates were incubated under the same incubation conditions for an additional $48 \mathrm{~h}$. To assess the effect of the RPV-field, cells were collected at 4 $\mathrm{h}, 24 \mathrm{~h}$ and $48 \mathrm{~h}$ respectively after the treatment, and subjected to the different assays. All experiments were performed in triplicate.

\section{Animal Care and Treatment}

Mice used in the experiments were purchased from Beijing Vital River Laboratory Animal Technology Co. Adult female C57BL/6] mice (8 months; $35 \pm 2 \mathrm{~g}$; specific pathogen-free) were maintained under a 12 -h dark/light cycle at about $22{ }^{\circ} \mathrm{C}$, and allowed food and drinking water ad libitum in the Animal Care Facility. Due to the limitation of the experiments, it is not allowed to treat animals with external Qi or Qi from RPV-field, so only Qi from Astragali Radix was used for the mice experiments. Animals were randomly divided into groups with three animals in each. In the treatment group, mice were intragastrically administered with Astragali Radix extract (dissolved in distilled water; $0.1 \mathrm{~g} / \mathrm{ml}$ ) at a daily dose of 0.20 $\mathrm{ml} / 10 \mathrm{~g}$ for 7 days. Control animals received water only. At $24 \mathrm{~h}$ after the last dose, the animals were anaesthetized with isoflurane gas, with the whole heart, liver, spleen, lung, kidney, brain, and skeletal muscle (hind limb) tissues excised and stored in liquid nitrogen. Minced tissue samples were homogenized in ice cold PBS (1/10, g/ $\mathrm{ml}$ ) with a Teflon-glass homogenizer. After that, the tissue extract was centrifuged at $3,000 \mathrm{~g}$ at $4^{\circ} \mathrm{C}$ for $5 \mathrm{~min}$ to remove cell debris and to obtain the final tissue homogenates which were subjected to measurement of telomere length, telomerase activity and TERT gene expression.

\section{Rotating Poynting Vector Generator}

For this work, we made our own RPV generator based on the basic principle of the Akimov Rotating Poynting Vector (RPV) generator [12-14], see (Figure 1). Generally speaking, the Poynting $\mathrm{S}$ vector is the flux density vector of the electromagnetic field, which can be defined by the following formula: $\mathrm{S}=[\mathrm{E} \times \mathrm{H}]$, where the $\mathrm{E}$ and $\mathrm{H}$ are the vectors of the electric and magnetic fields. Normally, the Poynting $\mathrm{S}$ vector propagates along a straight line

Table 1: Primers used in this study. in the direction of the electromagnetic wave. But in our specially designed generator, the Poynting vector S propagates in a spiral manner along the enamelled wire around the cone. The directions of the rotating S vector can be adjusted for left- and right-handed configurations by changing the direction of the signal. The magnet at the bottom of the generator is replaced by a stronger neodymium magnet. Between the copper cone and the copper coil, there is a plastic cone made using 3D printer, whose thickness is about $2 \mathrm{~mm}$. So, when the signal is applied to the copper coil, an electric field will be generated, whose direction is vertical to the inclined plane of the copper cone. Then the horizontal component of the electric field will be orthonormal to the vertical magnetic field. Therefore, a spiral RPV field will be generated around the plastic cone.

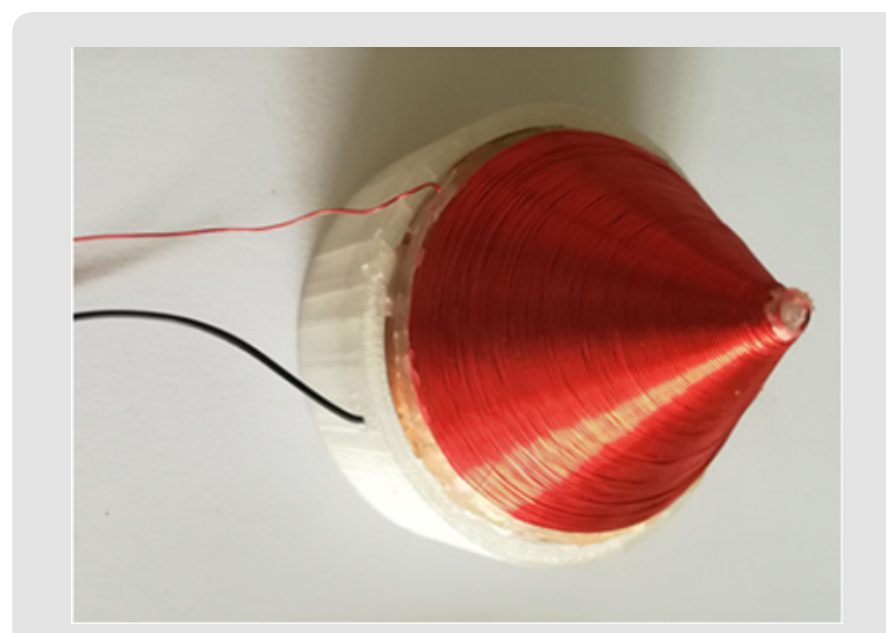

Figure 1: Rotating Poynting Vector (RPV) Generator. (The details of the equipment can be found in Ref [14]).

\section{Measurement of Telomere Length}

Total DNA was extracted from cell and tissue homogenates using the MiniBEST Universal Genomic DNA Extraction Kit (TaKaRa, 9765) according to the manufacturers' protocols. The telomere length was quantified by real-time PCR using TB Green® Premix Ex

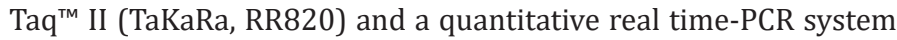
(ABI, QuantStudio3). The primers specific for telomere and nuclear gene B2M were listed in (Table 1). The relative length of telomere was obtained by normalization to B2M using the $2^{-\Delta \Delta \mathrm{Ct}}$ method.

\begin{tabular}{|c|c|c|}
\hline P rimers & Sequence & Accession \\
\hline TERT-Mouse-F1 & ACGGATATCTGGCCCTCTGT & AF051911.1 \\
\hline TERT-Mouse-R1 & ATTTGCCTGATGGGGTTGGT & \multirow{2}{*}{ NM_007393.5 } \\
\hline$\beta$-actin-Mouse-F1 & AGATCAAGATCATTGCTCCTCCT \\
\hline$\beta$-actin-Mouse-R1 & ACGCAGCTCAGTAACAGTCC & \multirow{2}{*}{ NC_000068.7 } \\
\hline Telo-Mouse-F2 & GGTTTTTGAGGGTGAGGGTGAGGGTGAGGGTGAGGGT \\
\hline Telo-Mouse-R2 & TCCCGACTATCCCTATCCCTATCCCTATCCCTATCCCTA \\
\hline mB2M-F1 & ATGGGAAGCCGAACATACTG \\
\hline
\end{tabular}




\section{Measurement of TERT Gene Expression}

Total RNA was extracted from M-1 cells using the MiniBEST Universal RNA Extraction Kit (TaKaRa, 9767) according to the manufacturers' protocols. RNA was then reverse transcribed into cDNA utilizing Reverse Transcriptase M-MLV (TaKaRa, 2641A). Gene expression was quantified by real-time PCR using TB Green $₫$ Premix Ex Taq ${ }^{\text {TM }}$ II (TaKaRa, RR820) and a quantitative real timePCR system (ABI, QuantStudio 3). The primers specific for TERT and $\beta$-actin were listed in (Table1). The relative abundance of each target was obtained by normalization to $\beta$-actin using the $2^{-\Delta \Delta \mathrm{Ct}}$ method.

\section{Measurement of Telomerase}

Telomerase activity was quantified in accordance with the protocol of the Mouse telomerase(TE)-ELISA assay kit (Product No.: JLC3717, Shanghai Jingkang Bioengineering Co., Ltd; China). The concentration of telomerase activity in the samples was determined by comparing the $\mathrm{OD}$ of the samples at $450 \mathrm{~nm}$ relative to the standard curve. Finally, telomerase activity in the cells was normalized to the total cell protein.

\section{Measurement of Protein}

The number of cells in the different experiment groups were evaluated by measuring total cell protein. Protein concentrations were determined with a protein assay kit (Product No.: P0006C; Beyotime, China) using bovine serum albumin as a standard $(0 \sim 1.5$ $\mathrm{mg} / \mathrm{ml}$ ). An $0.5 \mathrm{ml}$ aliquot of cell samples was centrifuged at 3000 $\mathrm{g}$ for $5 \mathrm{~min}$ to collect the cells. Then the cells were incubated with Triton $(0.1 \%)$ for $30 \mathrm{~min}$. After cell disruption, samples were centrifuged at $11,000 \mathrm{~g}$ for $5 \mathrm{~min}$ to remove cell residue. Finally, the optical density was measured at a wavelength of $595 \mathrm{~nm}$ using a using a microplate reader (Tecan, Switzerland).

\section{Statistical Analysis}

The values of different measurements were normalized to a respective mean control value from untreated samples and expressed as percent control. All data were expressed as mean standard deviation of the mean (SD). To statistically evaluate the biological effects of Qi, data were analysed by two-way analysis of variance (ANOVA) followed by Fisher's LSD multiple comparisons tests. GraphPad InStat software was used for the analysis, where $\mathrm{p}<0.05$ was considered to be statistically significant.

\section{Results}

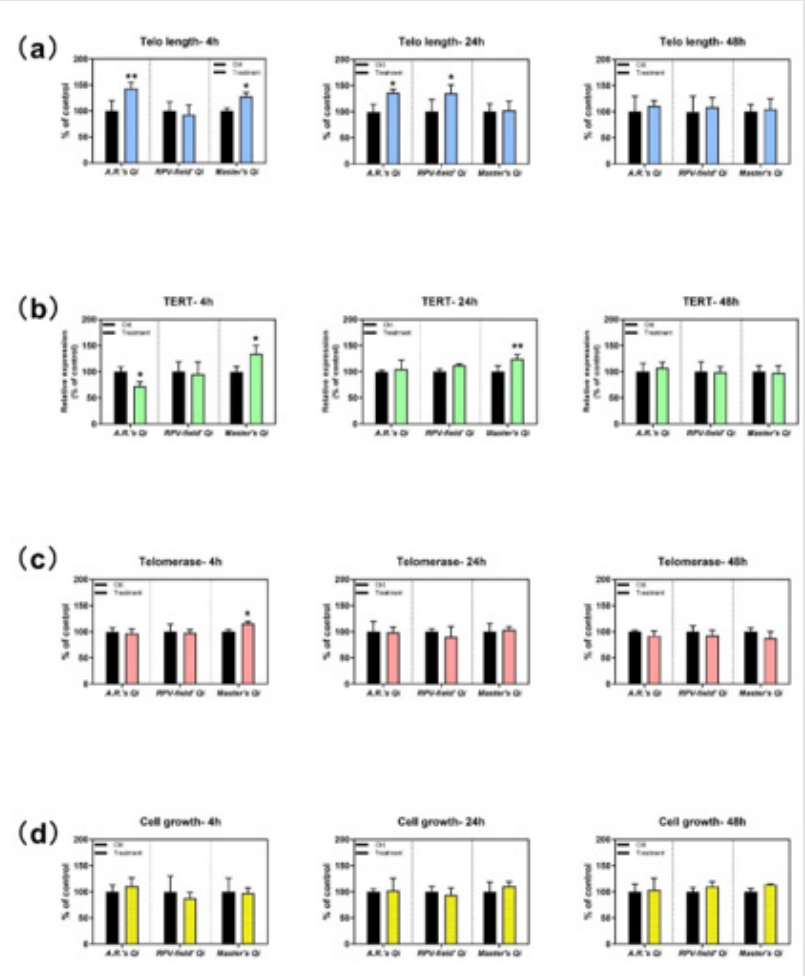

Figure 2: Effects of different sources of Qi on cells

(a) Telomere length;

(b) Telomerase;

(c) TERT gene expression;

(d) Cell growth. A.R.'s Qi: Qi from Qi-nourishing Chinese herbs Astragali Radix; RPV-field's Qi: Qi from RPV-field; Master's Qi: external Qi from Qigong master. Data were expressed as the relative percent with respect to the control group. ${ }^{*} \mathrm{P}<0.05$; **P $<0.01$. 
In terms of telomere length (as showed in (Figure 2a), the three different sources of Qi showed the ability to prolong telomere length at varying degrees. After the treatment with the Qi generated from Astragali Radix (A.R.), the telomere length was prolonged by $43 \%$ and $36 \%$ at $4 \mathrm{~h}$ and $24 \mathrm{~h}$ respectively, when compared with the control group. The Qigong mater's Qi prolonged the telomere length by $28 \%$ at $4 \mathrm{~h}$. The effect of the RPV-field on telomere length showed a $35 \%$ increase at $24 \mathrm{hr}$ but did not show any difference at 4 $\mathrm{h}$. However, at $48 \mathrm{~h}$ after the treatment, no matter what the sources of Qi, there was no difference between treatment and control group. Regulation of TERT gene expression was different under different sources of Qi. As shown in (Figure 2b), the master's Qi up-regulated TERT gene expression by $34 \%$ and $24 \%$ at $4 \mathrm{~h}$ and $24 \mathrm{~h}$ respectively, compared to the control group. But A.R.'s Qi down- regulated TERT gene expression by $28 \%$ at $4 \mathrm{~h}$, and there were no effects for the RPV-field treatment.

For telomerase activity (as showed in (Figure 2c), the change was relatively conservative, with a slight enhancement of $16 \%$ at $4 \mathrm{~h}$ after treatment with the master's Qi. In addition, the three sources of Qi did not show a significant effect on cell growth (as showed in (Figure 2d). As showed in (Figure 3a), A.R.'s Qi caused a significant increase in telomere length in most of the mouse tissues examined including heart, liver, spleen, and lung by $33 \%, 34 \%$, $32 \%, 33 \%$ respectively. However, there was no significant change in the kidney, brain or muscle tissue. (Figure $3 b$ ) shows that the treatment of A.R.'s Qi did not produce any significant changes in the weight of organs.

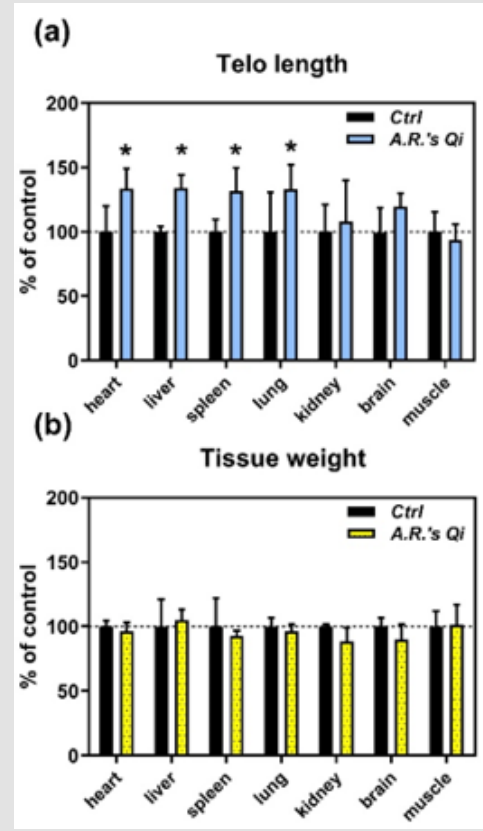

Figure 3: Effects of Qi from Chinese herbs Astragali Radix (A.R.) on various tissues of mice

(a) Telomere length;

(b) Tissue weight. Data were expressed as the relative percent relative to the control group. ${ }^{*} \mathrm{P}<0.05$; ${ }^{* *} \mathrm{P}<0.01$.

\section{Discussion}

Qi is neither a paranormal nor parapsychological phenomenon but is a normal phenomenon [15]. So the question remains what exactly is Qi? Its electromagnetic nature is established as demonstrated by published studies that weak EM emissions generated from the end of an acupuncture meridian where Qi leaves the body were detected [16-18]. However, according to Traditional Chinese medicine (TCM), Qi is much more than an electromagnetic field - it is the fundamental energy of the life force itself which sustains all human life activities. The ancient text "the Yellow Emperor's Inner Canon (Nei Jing)" stated that "Qi is the foundation of the human body". And another ancient text Jingyue Zhang's "Nei Jing" stated that "Human life depends on the Qi." with further elaborating that Qi promotes the growth and development of the human body, improves the physiological functions of each viscera and bowels, and adjusts the circulation of blood [19]. Enhancing Qi in the body, accomplished by a variety of different techniques in TCM, results in a healthy body including promoting the growth and development of the human body, promoting the physiological functions of organs, helping to control homeostasis, and providing warmth for the body, defending the body from external pathogens, holding the organs so as to prevent them from descending and so on $[15,20]$. Most of these studies used the technique of Qigong to increase the subjects' internal life force or Qi. Other studies used specific Chinese herbs, like A.R. , to enhance one's Qi.

TCM theory also states that Qi decreases as aging progresses [21]. Qi deficiency symptoms in the viscera lead to aging disease, and Qi deficiency in general has been proposed as the underlying mechanism of aging [22]. One experimental approach to studying the nature of Qi is to characterize the energy by virtue of its effects on biological systems. Although the difficulties in using modern western scientific methodology to study the eastern concept of Qi have been pointed out [23], several scientific studies have attempted to examine the effect of Qi on aging by using biochemical and biomedical methods. In their studies, the mechanism behind the effect of Qi on aging was attributed to reducing the production of reactive oxygen species (ROS) [24], regulation of mitochondrial function [25], improvement of gastro-enteric circulation [26], or enhancement of bone density as people age [27]. In this study, the measurement of telomeres was recognized as a valid scientific biomarker to evaluate the effects of $\mathrm{Qi}$ on aging. In western biomedical science, telomere shortening is recognized as one of the main biomarkers and causes of aging, especially in higher mammals and organisms [28].

Telomeres are a special DNA fragments at the end of chromosomes which are made up of many short simple repeated sequences. Telomere length is shortened with increased cell division and the ability of cell replication is restricted, thus offering a mechanism of aging [29-31]. Studies demonstrated that one of the key factors for the telomeres shortening is the decrease or loss of telomerase activity in cells [32]. Telomerase is a 
ribonucleoprotein complex which can take its own RNA component as template to replicate new DNA fragments adding to the end of telomeres to repair and maintain telomere length. Furthermore, telomerase activity mainly depends on the regulation of the telomerase reverse transcriptase gene (TERT) [33]. Therefore, telomere length, telomerase activity and TERT gene expression can be considered together as biomarkers of the aging process. Our results showed that all three sources of Qi prolonged the telomere length at the cellular level, albeit by different mechanisms. Only external Qi from a Qigong master stimulated all three biomarkers measured in the present study. The other two methods only stimulated one biomarker- telomere length. The Qi invigorating herb, A.R., enhanced telomere length both in-vitro and in-vivo. We speculate that controlling telomere length is one of the underlying mechanisms for Qi to slow down aging. In addition, the effect of Qi seems to have an acute stimulatory effect in less than 24 hours.

This finding is similar to a study conducted by Lee et al, which reported that external Qi transmitted from a Qigong master had a rapid effect on neutrophil superoxide generation but disappeared over time [34]. In addition, Qi from Astragali Radix showed viscera specific effects on telomere. The reason behind this phenomenon needs further research to illuminate the underlying mechanism of Qi on organs.

\section{Conclusion}

In conclusion, the results obtained from the present study indicate that Qi can prolong the telomere length for both cells cultured in vitro and different organs of mice. These findings implicate telomere lengthening as one potential mechanism for the effect of Qi on the retardation of aging. More importantly, Qi transmitted from Qigong master, Qi from Qi-invigorating Chinese herb, or the Qi mimicked by a physical field all produced the same effect to lengthen telomeres. These findings for the first time offer new experimental in-vitro and in-vivo data to link the eastern TCM concept of Qi to western medicine in general and aging biomarkers in specific.

\section{References}

1. Lee SC (2020) The Science of Torsion Field. Sancai Culture Co., LTD, China.

2. Wallace DC (2008) Mitochondria as chi. Genetics 179(2): 727-735.

3. Lin F, Guo LL, Wang J (2014) Discussion on The Relationship of Mitochondrion and Qi. Chinese J Basic Med TCM 34(8): 903-906.

4. Han JX, Han Y (2010) The theory of the material basis of Chinese medicine Qi is the electromagnetic (quantum) field radiated by the body. J Shandong Univ Tradit Chin Med 34(6): 474-479.

5. Lin JS, Zhang LX (2002) A New Explanation of Qi's Materiality from Quantum Theory. Study Journal of Traditional Chinese Medicine (3): 325-327.

6. Gao P, Kernbach S (2018) External Qi and Torsion Field. Somatic Science Conference, China.

7. Lee SC, Sun CL, Shen JC, Hou JR (1999) Research on Consciousness Regulation on Resurrection of Flowers. Chinese Journal of Somatic Science 9(2): 75-77.
8. Yuan TZ, Ormonde CFG, Kudlacek ST, Sameeran Kunche, Joshua N Smith, et al. (2015) Shear-stress-mediated refolding of proteins from aggregates and inclusion bodies. Chembiochem 16(3): 393-396.

9. Meyerson M (2000) Role of telomerase in normal and cancer cells. J Clin Oncol 18(13): 2626-2634.

10. Hartwig FP, Bertoldi D, Larangeira M, Wagner MS (2014) Up-regulating telomerase and tumor suppressors: Focusing on anti-aging interventions at the population level. Aging Dis 5(1): 17-27.

11. De Jesus BB, Schneeberger K, Vera E, Tejera A, Harley CB, et al. (2011) The telomerase activator TA-65 elongates short telomeres and increases health span of adult/old mice without increasing cancer incidence. Aging Cell 10(4): 604-621.

12. Akimov A, Petrovsky B, Tarasenko V (1995) Structure and construction of torsion generators, pre-pring N52 (rus) M, MNTC VENT.

13. Kernbach S (2017) Tests of the circular Poynting vector emitter in static E/H fields. International Journal of Unconventional Science pp. 23-39.

14. Gao P (2018) Detection of Torsion Field Based on Measuring the Dark Current of Silicon Photodiode. International Journal of Unconventional Science (E3): 3-16.

15. Li XT, Zhao J (2012) An approach to the nature of Qi in TCM-Qi and bioenergy. Recent Advances in Theories and Practice of Chinese Medicine 79-108.

16. Majid A, Rouh MA (2018) Qi before and after production; Basic concept of life. Int J Complement Alt Med 11(2): 139-143.

17. Yang M, Van Wijk E, Pang J (2019) A Bridge of Light: Toward Chinese and Western Medicine Perspectives Through Ultraweak Photon Emissions. Global Advances in Health and Medicine 8: 2164956119855930.

18. Park SH, Kim J, Koo TH (2009) Magneto-acupuncture stimuli effects on ultraweak photon emission from hands of healthy persons. Journal of Acupuncture and Meridian Studies 2(1): 40-48.

19. Sun GR (2016) Basic Theory of Chinese Medicine. China Traditional Chinese Medicine Press, China.

20. Lin F, Guo L, Wang J (2014) Discussion on The Relationship of Mitochondrion and Qi. Chinese J Basic Med TCM 34(8): 903-906.

21. Zhang TJ, Chen CQ (2007) Modern research and application of anti-aging and anti-fatigue Chinese medicine. China Traditional Chinese Medicine Press, China.

22. Zhang ML, Zhang LT, Qiu XF, Zhou AF (2001) Discussion on The Relationship of Mitochondrion and Qi. Chinese J Basic Med TCM 7(04): 60-61.

23. Ohnishi ST, Ohnishi T (2009) Philosophy, psychology, physics, and practice of ki. Evidence-Based Complementary and Alternative Medicine 6(2): 175-183.

24. Ohnishi T, Ohnishi T, Nishino K, Tsurusaki Y, Yamaguchi M (2005) Growth inhibition of cultured human liver carcinoma cells by Ki-energy (life-energy): Scientific evidence for Ki-effects on cancer cells. EvidenceBased Complementary and Alternative Medicine 2(3): 387-393.

25. Ohnishi T, Ohnishi T (2006) The Nishino breathing method and ki-energy (life-energy): A challenge to traditional scientific thinking. EvidenceBased Complementary and Alternative Medicine 3(2): 191-200.

26. Shih HC, Chang KH, Chen FL, CM Chen, SC Chen, et al. (2000) Anti-aging effects of the traditional Chinese medicine bu-zhong-yi-qi-tang in mice. The American journal of Chinese medicine 28(1): 77-86.

27. Nishino K (2006) The Dictionary of Respiration. Tokyo: Asakura Book Publishing Co., Japan.

28. Vidaček NŠ, Ćukušić A, Ivanković M, Hrvoje Fulgosi, Miljenko Huzak, et al. (2010) Abrupt telomere shortening in normal human fibroblasts. Experimental gerontology 45(3): 235-242.

29. Harley CB, Futcher AB, Greider CW (1990) Telomeres shorten during ageing of human fibroblasts. Nature 345(6274): 458-460. 
30. Blackburn EH (1991) Structure and function of telomeres. Nature 350(6319): 569-573

31. Blasco MA, Lee HW, Hande MP, E Samper, P M Lansdorp, et al. (1997) Telomere shortening and tumor formation by mouse cells lacking telomerase RNA. Cell 91(1): 25-34.

32. Gilley D, Herbert BS, Huda N, Tanaka H, Reed T (2008) Factors impacting human telomere homeostasis and age-related disease. Mechanisms of ageing and development 129(1-2): 27-34.

ISSN: 2574-1241

DOI: $10.26717 /$ BJSTR.2021.33.005374

Xuemei Bai. Biomed J Sci \& Tech Res

(C) (i) This work is licensed under Creative Commons Attribution 4.0 License

Submission Link: https://biomedres.us/submit-manuscript.php
33. Guo XH, Liu N (2018) The New Biological Functions of Telomerase Reverse Transcriptase. Chinese Journal of Biochemistry and Molecular Biology 34(9): 927-934

34. Lee MS, Jeong SM, Jang HS, Ryu H, Moon SR (2003) Effects of in vitro and in vivo qi-therapy on neutrophil superoxide generation in healthy male subjects. The American journal of Chinese medicine 31(4): 623-628.

$\begin{array}{ll}\text { BIOMEDICAL } & \text { Assets of Publishing with us } \\ \text { RESEARCHES } & \text { - Global archiving of articles } \\ \text { - Immediate, unrestricted online access } & \text { - Rigorous Peer Review Process } \\ & \text { - Authors Retain Copyrights } \\ & \end{array}$

